\title{
Studies of exotic nuclei with advanced radiation detectors
}

\author{
Zsolt Podolyák \\ Department of Physics, University of Surrey, Guildford, GU2 7XH, UK
}

\begin{abstract}
Contemporary key nuclear physics questions are introduced. The role of radiation detection in the study of exotic nuclei is illustrated with examples related to NuSTAR at the FAIR facility. The discussed detection systems include: Si-tracker for light charged particle detection, the AGATA gamma-ray tracking detector, diamond detectors for heavy ion measurements, the AIDA implantation and decay detector, and the $\mathrm{LaBr}_{3}(\mathrm{Ce})$ fast-timing array. Due to technology transfer, applications related to radiation physics are expected to benefit from these developments.
\end{abstract}

Keywords: Nuclear physics, detectors

\section{Introduction}

The vast majority, over $99.9 \%$, of the mass in the visible universe is concentrated in atomic nuclei. Currently, out of the around 7000 possible bound nuclei, some experimental information is known on less than 4000 nuclear species. In particular we have information mainly on stable nuclei and those on the proton rich side of the valley of stability (see figure 1). Although various models of nuclear matter can adequately describe some of the properties of many of these known nuclei, they have little in the way of predictive power. For example the mass of the nucleus is one of its simplest gross properties, but we are unable to predict its value accurately for an unknown nucleus. Similarly, the neutron emission limit, or drip-line, cannot be predicted (for the case of the tin isotopes see (Patra et al., 2005)). Different mass models and mass parameterisations are working well for nuclei for which the mass was actually measured. However they differ hugely for nuclei where no experimental information is available (see figure 2). The reason for this is 
that a number of parameters used in the theories, which come from known nuclei, have to be extrapolated in a poorly defined manner.

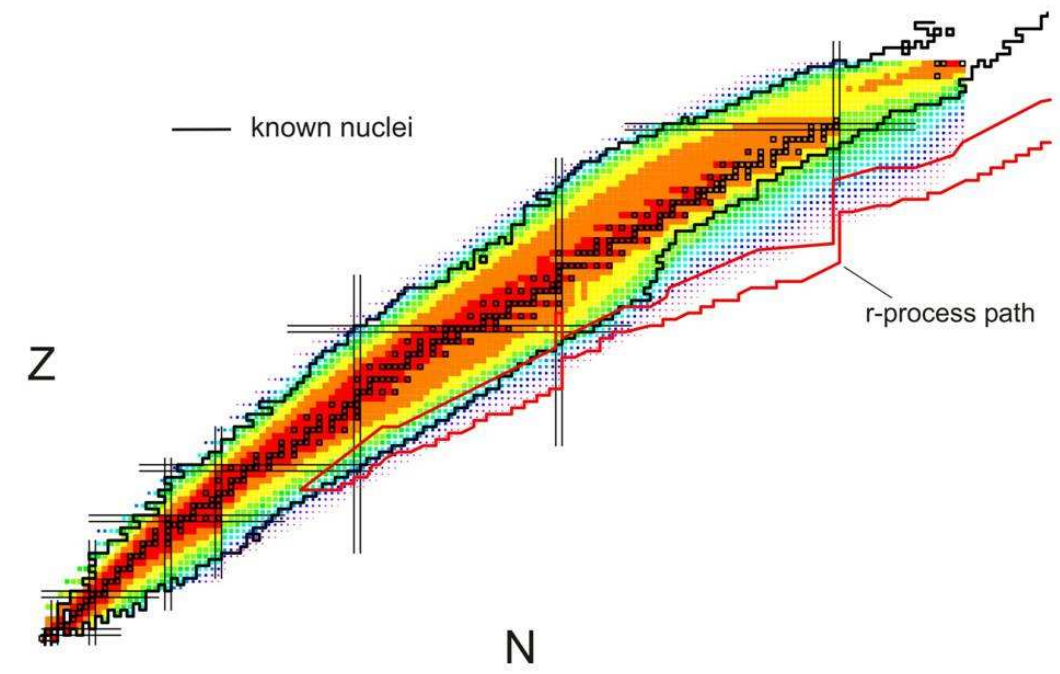

Figure 1: Nuclide chart showing the nuclei already studied (solid line). The dotted area shows the nuclei which will be produced by the future radioactive beam facility at FAIR; several of them are on the r-process path.

Experimentally, information about nuclei are obtained by detecting and analysing the properties of the radiation leaving the system. Gamma-ray and conversion electron detection give information about excited states. In addition to the energy measurements, angular distributions, polarisations, lifetimes etc. can be determined and used to infer the properties/wave-functions of the nuclear states. Some excited states and the majority of the groundstates (with the exception of the stable nuclei) will decay via alpha decay, beta decay, proton decay, two-proton decay, or possibly neutron decay. In addition, different reactions can be used to produce exotic nuclei and by measuring the properties of incoming and outgoing particles information about the nucleus of interest can be obtained.

Currently nuclear physics is being revolutionised by the new radioactive ion beam accelerator facilities. The biggest European Facility, the GSI-FAIR complex is being built in Darmstadt, Germany (Krücken, 2005). The aim of 


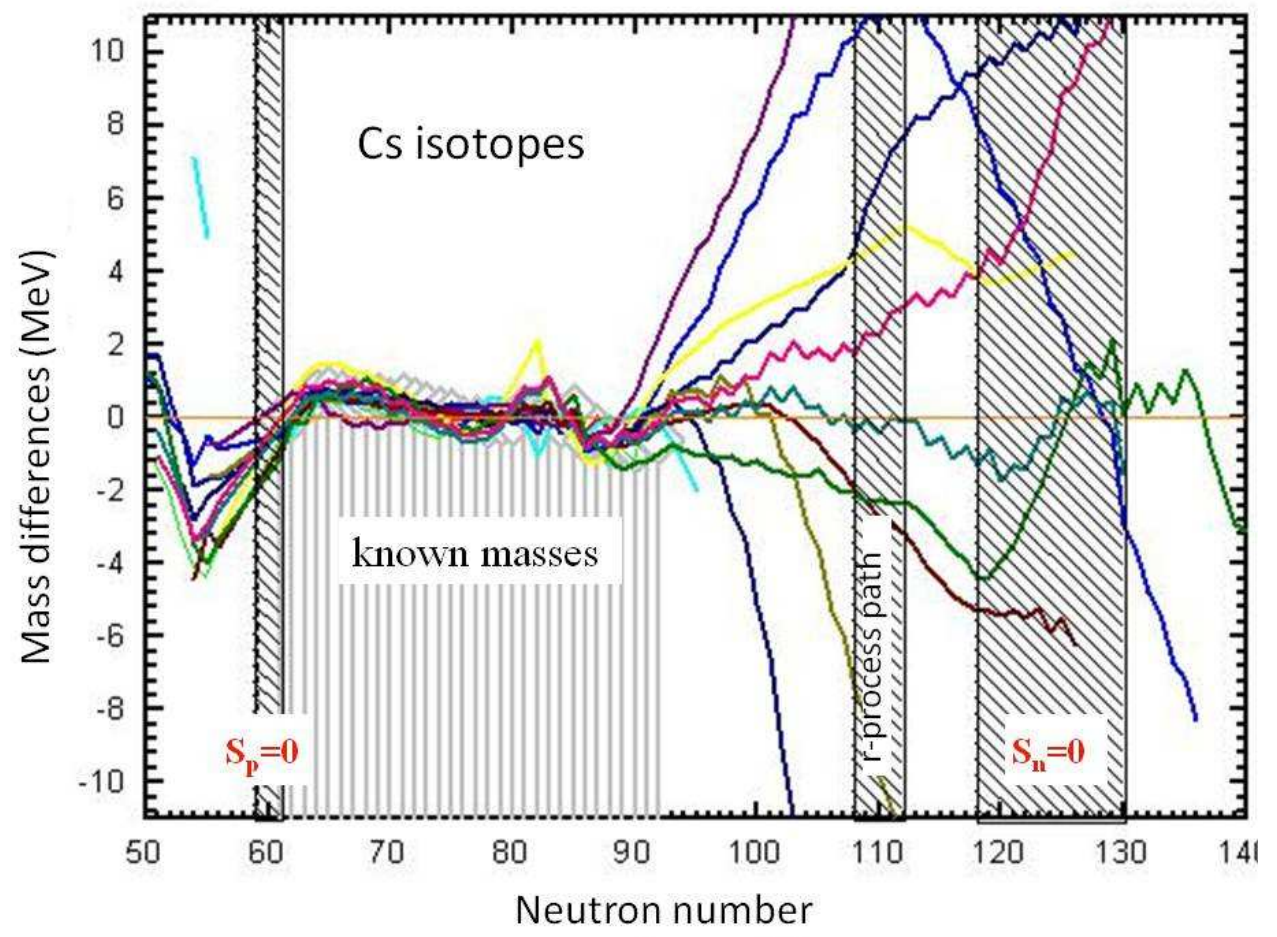

Figure 2: Mass differences predicted by different mass models and parameterisations for Cs isotopes $(\mathrm{Z}=55)$. The predicted positions of the proton drip-line, r-process path and the neutron drip-line are indicated.

the Nuclear Structure, Astrophysics and Reaction (NuSTAR) collaboration at FAIR is to study exotic nuclei, mainly on the neutron-rich side (see figure 1). NuSTAR (Rubio, 2006) will be able to study the properties of nuclei which previously could not be produced.

The present paper will discuss the use of radiation detectors in fundamental nuclear physics. The status of the exotic nuclei studies will be illustrated via examples related to NuSTAR. State-of-the-art radiation detector systems developed for charged particles and electromagnetic radiation will be discussed. 


\section{Key physics questions in nuclear physics}

Key physics questions in nuclear physics are: (i) what is the nature of nuclear matter?; (ii) how were the heavy elements synthesised?

(i) the nature of nuclear matter: Nuclei are formed by protons and neutrons. Even if the interaction between the nucleons within the nucleus were known, such a many-body problem cannot be solved. The problem is even more difficult, as the form of the nucleus-nucleus interaction is not known exactly. Additional complication is brought in by the non-elementary nature of the nucleons. In order to deal with this problem, three-body forces are also introduced. Considering all interactions between the constituents of the nucleus, the problem cannot be solved mathematically. Therefore, simplifications are made, and different models are used. Some models describe some properties of some nuclei. However single unified model of nuclei does not exist so far. Questions as how many neutrons can be added to an element before it falls apart (where the neutron drip-line is) or how the simplicity of collective states arise from the complexity of many individual nuclei cannot be satisfactory answered at the moment.

One crucial question in nuclear physics is how the quantum orbitals evolve as the number of protons and neutrons change in the system. The nuclear potential (the potential energy experienced by a nucleon, generated from its interactions with the other nucleons in the nucleus) is expected to take the form of the nuclear density. Close to stability, this has relatively well defined edge (surface), as shown in figure 3. For neutron-rich nuclei, with high neutron/proton ratio, the neutron matter radius is larger than the proton radius, therefore the nuclear potential has a more diffused surface. As a consequence, the ordering of the nuclear orbitals can change drastically in neutron-rich nuclei (see figure 3). In extreme cases, traditional magic numbers can disappear and new ones can emerge. Such changes were already observed for light nuclei, and are predicted for heavy ones. The evolution of orbitals and shell gap have obvious consequences on nucleosynthesis, as discussed below.

(ii) production of heavy elements: Half of the nuclei heavier than iron are believed to be synthesised in the rapid neutron-capture process, the so called r-process (Arnould et al., 2007). No comprehensive model for the r-process exists. The understanding of the observed abundance pattern in the solar system and other stars requires detailed knowledge of the r-process waiting point nuclei (Nishimura et al., 2011). These are far from stability 


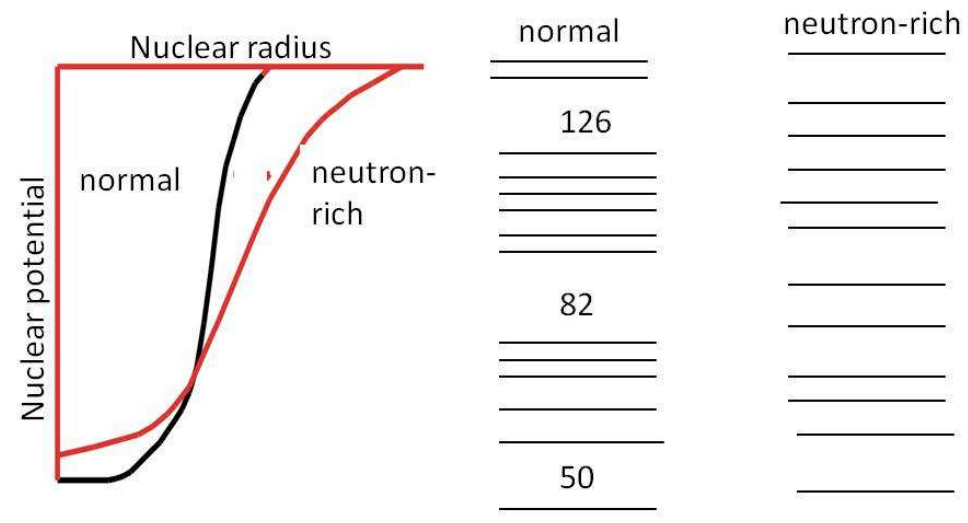

Figure 3: The nuclear potential for normal matter as well as for neutron-rich matter is shown on the left. The corresponding change in the ordering of nuclear orbitals, are illustrated on the right hand side. The traditional magic numbers are indicated.

and at the moment very few of them can be studied experimentally. The NuSTAR facility will be unique in producing heavy r-process path nuclei (e.g. the heaviest of the $\mathrm{N}=126$ r-process waiting point nuclei), related to the increased abundances in the A 195 mass region (see figure 1). The nuclear physics information is one of the main ingredients in nucleosynthesis, and its knowledge is needed to constrain the stellar evolution models (such as constraints on astrophysical conditions governing the neutron density, its time dependence, and the amount of ejected material) (Cowan, 2003).

Another open nucleosynthesis question is related to elements around SrY-Zr. The abundances of these elements are poorly understood, both in the solar system and in old stars. It was postulated that they are partly synthesised in the so called light-element primary process (LEPP). The nature of this process as well as the possible sites for it are debated ( $\nu$ p process, additional r-process or s-process component) (Montes et al., 2007). The study of nuclei in this region, from the proton drip-line up to extremely neutron-rich isotopes, might help to shed more light on this mysterious process. 
There is no single experiment which can answer the above physics questions. Rather, a number of nuclei have to be studied, using different techniques.

\section{Detector systems and collaborations at NuSTAR}

NuSTAR (Nuclear Structure, Astrophysics and Reactions) is an umbrella collaboration. It comprises nine different collaborations based around stateof-the-art detector systems. Here we discuss some examples of equipments used in three of them: $\mathrm{R}^{3} \mathrm{~B}$ (Reactions with Relativistic Radioactive Beams), HISPEC (High-resolution in-flight spectroscopy) and DESPEC (Decay Spectroscopy). Examples of detector systems are presented: for proton detection (silicon tracker for $\mathrm{R}^{3} \mathrm{~B}$ ), next generation $\gamma$-ray detector (the AGATA $\gamma$-ray tracking array used at HISPEC), large area diamond detectors (LYCCA for HISPEC), implantation and decay detector (AIDA for DESPEC), fast scintillation array (fast-timing array for DESPEC). These examples were selected as they are fully or partially built by the UK.

\subsection{Si-tracker for $R^{3} B$}

The $\mathrm{R}^{3} \mathrm{~B}$ collaboration aims to use reactions at relativistic energies to study the properties of exotic nuclei. Its strengths lies in its full kinematics measurements. Therefore both the incoming particle, as well as the outgoing particles has to be detected, identified, tracked and their energies measured. The setup comprises a large number of detectors, for heavy charged particles, light charged particles, neutrons and photons. Here we discuss in some detail the Si tracker which is being built in Daresbury-Liverpool-EdinburghBirmingham-Surrey collaboration (Lemmon, 2012). It is aimed to the detection of light charged particles, mainly protons, knocked out from the target. It consists of three layers of $\mathrm{Si}$, with thicknesses of $100 \mu \mathrm{m}$ for the inner layer and $300 \mu \mathrm{m}$ for the two outer layers (see figure 4). The Si are double-sided silicon strip detectors with huge number of pixels, allowing a high precision tracking. In addition the proton energies have to be measured. Due to the large number of electronics channels required, an ASIC (Application Specific Integrated Circuit) is being developed.

\subsection{Examples of detectors used at HISPEC}

The experimental setup at HISPEC comprises a large number of detectors. The incoming radioactive beams will be tracked and their trajectory 


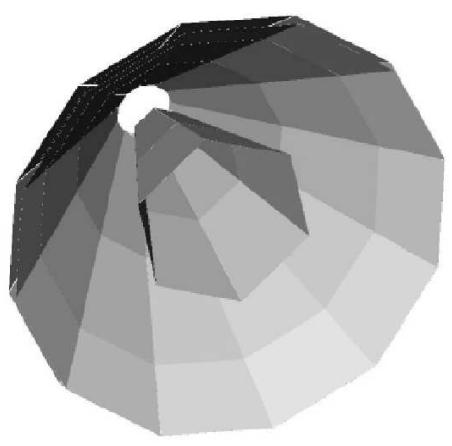

Figure 4: Conceptual design of the Si-tracker (Lemmon, 2012). It consist of three highly pixalleted Si layers. The second and third layers are close to each other.

and energy measured. They will impinge on a secondary target where they will be excited via Coulomb interaction or they will produce other species via nuclear reactions. The radiation from the excited nuclei will be detected with high-resolution $\gamma$-ray, and possible charged-particle detector arrays. The outgoing beam-like particles will also have to be tracked and identified. Tracking is essential for Doppler correction of the detected $\gamma$ rays, too.

The AGATA gamma-ray tracking array (Akkuyon et al., 2012) is at the heart of the HISPEC project. AGATA is developed by a dedicated European collaboration. A similar system, called GRETA, is being built in parallel in the USA (Lee et al., 2004). Traditional HPGe crystals measure the total energy deposited in the crystal. In contrast, Ge tracking detectors are able to provide information about the energy and position of each individual interaction between photon and the Ge. This is done by the use of segmentation and pulse shape analysis. Each AGATA Ge crystal is electrically segmented in 36 pieces, therefore individual signals are obtained from each segment. In addition, pulse shape analysis of each signal is performed, using fast digitisers. By analysing the shape of the real and induced signals, the position of the interaction point, $\mathrm{x}, \mathrm{y}, \mathrm{z}$, as well as the interaction energy can be determined for each interaction (see figure 5). Having this information, the track 
of the individual gamma-rays can be reconstructed, considering the laws of Compton scattering and pair production (see figure 6).

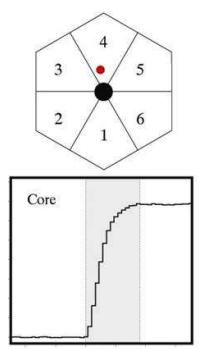

Time

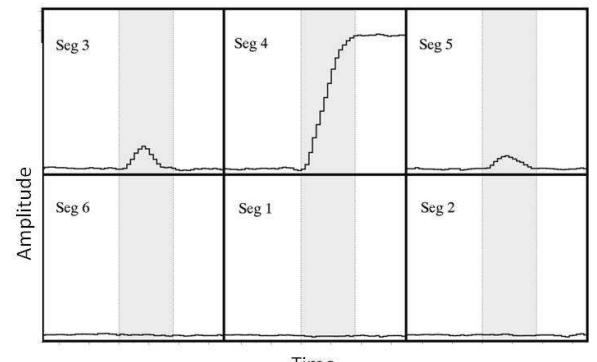

Time

Figure 5: The principle of pulse-shape analysis as applied in the case of the AGATA gamma-ray tracking array (Akkuyon et al., 2012). By digitising and examining the shape of the pulses in the segment where the interaction happened as well as the induced pulses in the neighbouring segments, the position of the interaction can be determined.

According to Monte Carlo simulations (Farnea et al., 2010), the full AGATA array, covering $4 \pi$ solid angle and consisting of 180 Ge crytals will have a full energy efficiency of $43 \%$ at $1 \mathrm{MeV}$. In contrast, $4 \pi$ arrays using traditional Ge detectors and BGO anti-Compton shields can achieve about $10 \%$ full energy peak efficiency (Simpson, 1997). In tracking detectors the position of the first interaction is determined with a precision in the order of $2 \mathrm{~mm}$. Consequently much more precise Doppler correction can be performed than in the case of the traditional Ge detectors, further improving the resolving power of tracking detectors. In fast-beam experiments, as performed at HISPEC, this is the main advantage of using the AGATA demonstrator.

AGATA is designed to be used at several laboratories. After experiments at the Legnaro National Laboratories (Italy), presently is at GSI, Darmstadt (Germany). At GSI it is used in experiments using radioactive beams at energies of $\mathrm{E} / \mathrm{A}=100-300 \mathrm{MeV}$. The present experimental campaign, running in years 2012-2014, is the precursor of the future HISPEC project.

At HISPEC, the identification of the reaction product needs precise velocity measurements. Different type of detectors are considered (LYCCA, 


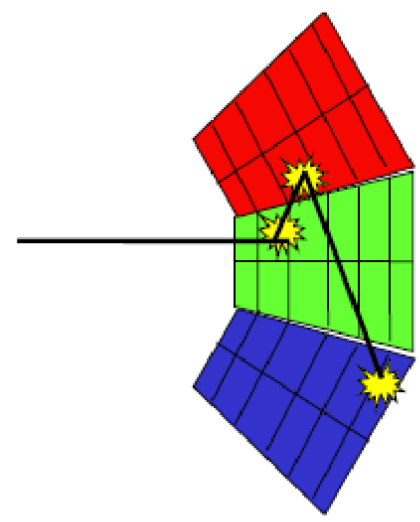

Figure 6: The principle of gamma-ray tracking (Akkuyon et al., 2012). Considering the rules of Compton scattering and pair production, one can determine with high probability whether interactions belong to the same gamma ray, as in the figure, or they originate from different photons.

2012; Hoischen et al., 2011), one of them being the use of diamond. Large area polycrystalline time-of-flight diamond detectors are being developed in York-Surrey collaboration. Preliminary results were published (Schirru et al., 2012). Diamond has the prospect of being used as an active target in the future, providing at the same time positon and timing information.

\subsection{Examples of decay spectroscopy detectors at DESPEC}

Decay spectroscopy is very sensitive method, providing information on very exotic nuclei. One recent example is the study of the $\beta$-decay of the doubly magic ${ }^{100} \mathrm{Sn}$ nucleus, produced at a rate of only 1 ion/hour (Hinke et al., 2012). Within the DESPEC project (Rubio, 2006) radioactive nuclei are implanted in a multi-layer highly segmented $\mathrm{Si}$ detector system, called AIDA (AIDA, 2012) (see figure 7). The same detectors will detect the charged particle emitted in the decay of the implanted nucleus. The main challenge of such a system is due to the need to be able to measure the energy of the implanted ion (in order of several $\mathrm{GeV}$ ), and shortly after the much lower energy of the decaying charged particle. In the case of internal decays via conversion electrons, these energies can be below $100 \mathrm{keV}$. This problem was solved by the development of an ASIC (Application Specific Integrated Circuit), enabling the measurement of high and low-energy particles 
detected in the same pixel. The AIDA implantation and decay detector was already built, in Edinburgh-Daresbury-Liverpool collaboration.

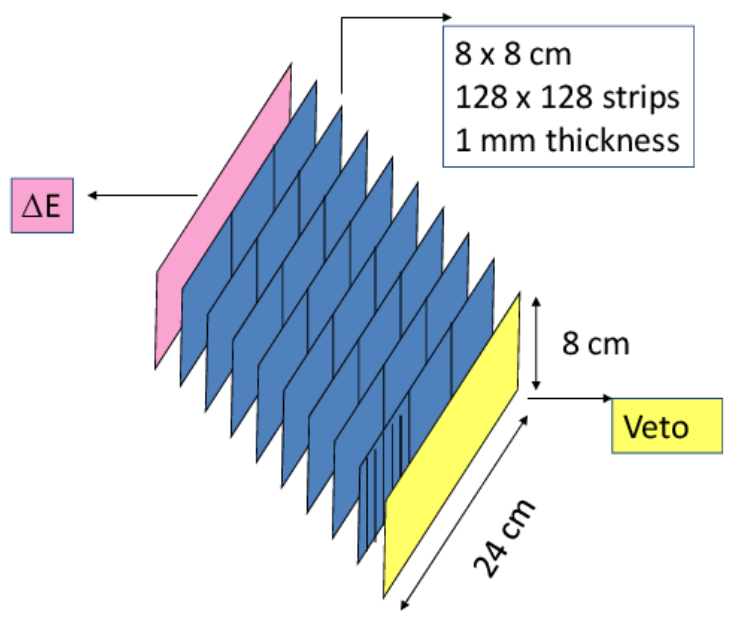

Figure 7: Schematic view showing the basic characteristics of the AIDA (AIDA, 2012) implantation and decay detector.

$\mathrm{LaBr}_{3}(\mathrm{Ce})$ detectors were developed relatively recently. These scintillation detectors have much better energy resolution, about $3 \%$ at $662 \mathrm{keV}$, than traditional scintillation materials. In addition, they are very fast, a property which can be exploited to measure lifetimes of excited states in the 100 ps - $10 \mathrm{~ns}$ region (Marginean et al., 2010). At DESPEC a fast-timing array is under construction (Regan, 2012). The fast-timing array will surround the AIDA decay detector, with a possible configuration (Roberts, 2012) shown in figure 8.

\section{Conclusions and outlook}

The field of fundamental nuclear physics is being revolutionised by the appearance of the next generation radioactive beam facilities. Consequently 


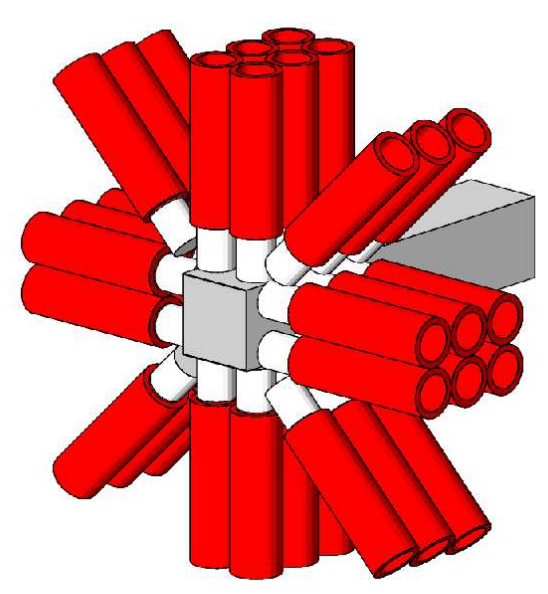

Figure 8: A possible arrangement of the $\operatorname{LaBr}_{3}(\mathrm{Ce})$ detectors (Roberts, 2012) around the AIDA decay detector (AIDA, 2012), planned to be used within the DESPEC collaboration. The white cylinders represent the scintillation crystals, with the dark cylinders being the photo-multiplier tubes.

a large number of previously unreachable nuclear systems will be studied, which in turn it will results in a better understanding of fundamental science questions such as 'how were/are heavy elements synthesised?'. In order to optimise the exploitation of these facilities costing hundreds of millions of Euros/Dollars, more sophisticated, higher efficiency radiation detectors systems are developed and built.

Several radiation detector systems used in the field of fundamental nuclear research were introduced. The presented examples will be used at the future FAIR and the existing GSI facilities within the NuSTAR collaboration. Similar detector systems are being built for other international facilities as well. The developments made, the technologies used, are being introduced into different fields of radiation physics and other related areas. For example, the good energy-resolution $\mathrm{LaBr}_{3}(\mathrm{Ce})$ scintillation detectors are already widely used as portable $\gamma$-ray detectors, replacing HPGe detectors. The same material, due to its very good timing properties, is expected to be used 
in time-of-flight positron emission tomography (TOF PET). Silicon has the prospect of being used in radiology, providing measurements of charged particles with very high position resolution. Tracking Ge detectors can be used for imaging (Schmidt et al., 2001), for example to localise radiation sources or used in scanning devices.

The detectors introduced here are developed by collaborations consisting of a large number of people. Although their names cannot be listed here due to space constraint, their work is acknowledged. This work is supported by $\mathrm{STFC}(\mathrm{UK})$.

\section{References}

AIDA webpage, 2012. www2.ph.ed.ac.uk/ td/AIDA/welcome.html [Accessed $1 / 10 / 2012]$

Arnould, M., Goriely, S., Takahashi, K., 2007. The r-process of stellar nucleosynthesis: Astrophysics and nuclear physics achievements and mysteries. Physics Reports 450, 97-213.

Akkuyon, S., et al., 2012. AGATA Advanced GAmma Tracking Array. Nucl. Instrum. and Meth. A 668, 26-58.

Cowan, J., 2003. Elements of Surprise. Nature 423, 29.

Farnea, E. et al. 2010. Conceptual desing and Monte Carlo simulations of the AGATA array. Nucl. Intrum. Meth. A 621, 331-343.

Hinke, C.B. et al. 2012. Superallowed Gamow-Teller decay of doubly magic nucleus ${ }^{100} \mathrm{Sn}$. Nature 486, 341-345.

Hoischen, R. et al. 2011. Fast timing with plastic scintillators for in-beam heavy-ion spectroscopy. Nucl. Instrum. Meth. A 654, 354-360.

Krücken, R., 2005. The NuSTAR facility at FAIR. J. Phys. G 31, S1807S1811.

Lee, I.Y. et al. 2004. GRETINA: A gamma ray energy tracking array. Nuclear Physics A 746, 255-259.

Lemmon, R., 2012. Private communication. 
LYCCA webpage, 2012. http://www.nuclear.lu.se/fileadmin/nuclear/Grundlaeggande/lyccaTDR.pdf [Accessed 1/10/2012]

Marginean, N. et al. 2010. In-beam measurements of sub-nanosecond nuclear lifetimes with a mixed array of $\mathrm{HPGe}$ and $\mathrm{LaBr}_{3}$ :Ce detectors. Eur. Phys. J. A46, 329-336.

Montes, F. et al. 2007. Nucleosynthesis in the Early Galaxy. Ap. J. 671, 1685-1695.

Nishimura, S. et al. 2011. $\beta$-Decay Half-Lives of Very Neutron-Rich Kr to Tc isotopes on the Boundary of the r-Process Path: An Indication of Fast r-Matter Flow. Phys. Rev. Lett. 106, 052502 pp.1-5.

Patra, S.K., Arumugam, P., Satpathy, L., 2005. Consistency of nuclear mass formulae. arXiv:nucl-th/0504063v1

Podolyák, Zs., 2006. The High-Resolution In-Flight Spectroscopy (HISPEC) project at FAIR. Int. J. of Mod. Phys. E15, 1967-1977.

Regan, P.H., 2012. From RISING to the DESPEC fast-timing project within NUSTAR at FAIR: Sub-nanosecond nuclear timing spectroscopy with $\mathrm{LaBr}_{3}$ scintillators. Appl. Rad. and Iso. 70, 1125-1130.

Roberts, O., 2012. Private communication.

Rubio, B., Nilsson, T., 2006. NuSTAR. Nucl. Phys. News 16, 9-14.

Rubio, B., 2006. Decay spectroscopy (DESPEC) at the new Fair-Nustar facility. Int. J. of Mod. Phys. E15, 1979-1988.

Simpson, J., 1997. The Euroball Spectrometer. Zeit. Phys. A 358, 139.

Schirru, F. et al. 2012. Development of large area polycrystalline diamond detectors for fast timing application of high-energy heavy-ion beams. J. of Instr. 7, P05005. pp.1-12.

Schmidt, G.J. et al. 2001. Gamma-ray Compton camera imaging with a segmented HPGe. Nucl. Instr. Meth. A 459, 565-576. 